\title{
Ablación y Neuromodulación en Tormenta Arrítmica. Serie de 4 casos y revisión de la literatura
}

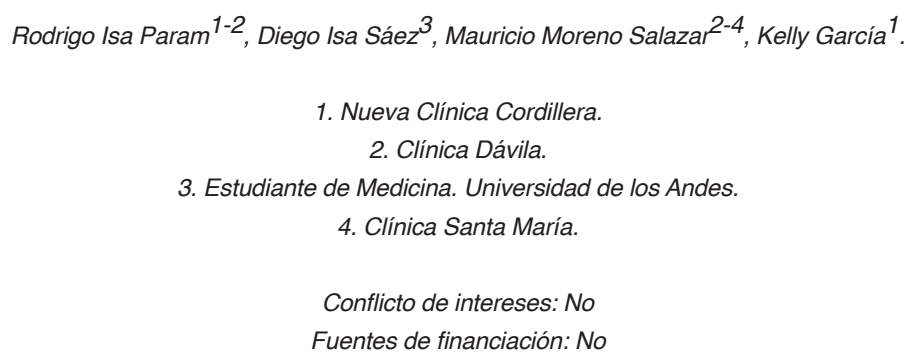

Se presenta una serie de 4 casos clínicos de pacientes con y sin cardiopatía estructural, que tuvieron uno o más episodios de tormenta arrítmica. Se describen los tratamientos con sus resultados y una revisión bibliográfica con los avances en el tema más allá de la ablación con catéter.

Palabras clave: Tormenta arrítmica; taquicardia ventricular; ablación; ganglio estrellado; simpatectomía: arritmia.

\section{Ablation and Neuromodulation in Arrhythmic Storm. A report of 4 cases and literature review}

We present 4 clinical cases of patients with and without structural heart disease, who had one or more episodes of arrhythmic storm. Treatments, results and a bibliographic review with advances beyond catheter ablation are described.

Keywords: arrhythmic storm; ventricular tachycardia; ablation; stellate ganglion; sympathectomy; arrhythmia. 


\section{Introducción:}

El término "Tormenta Arrítmica" (TA) se usa para describir un período de grave inestabilidad eléctrica, que se manifiesta por arritmias ventriculares recurrentes. Su definición ha evolucionado con el uso generalizado de los desfibriladores implantables, siendo la más aceptada, la

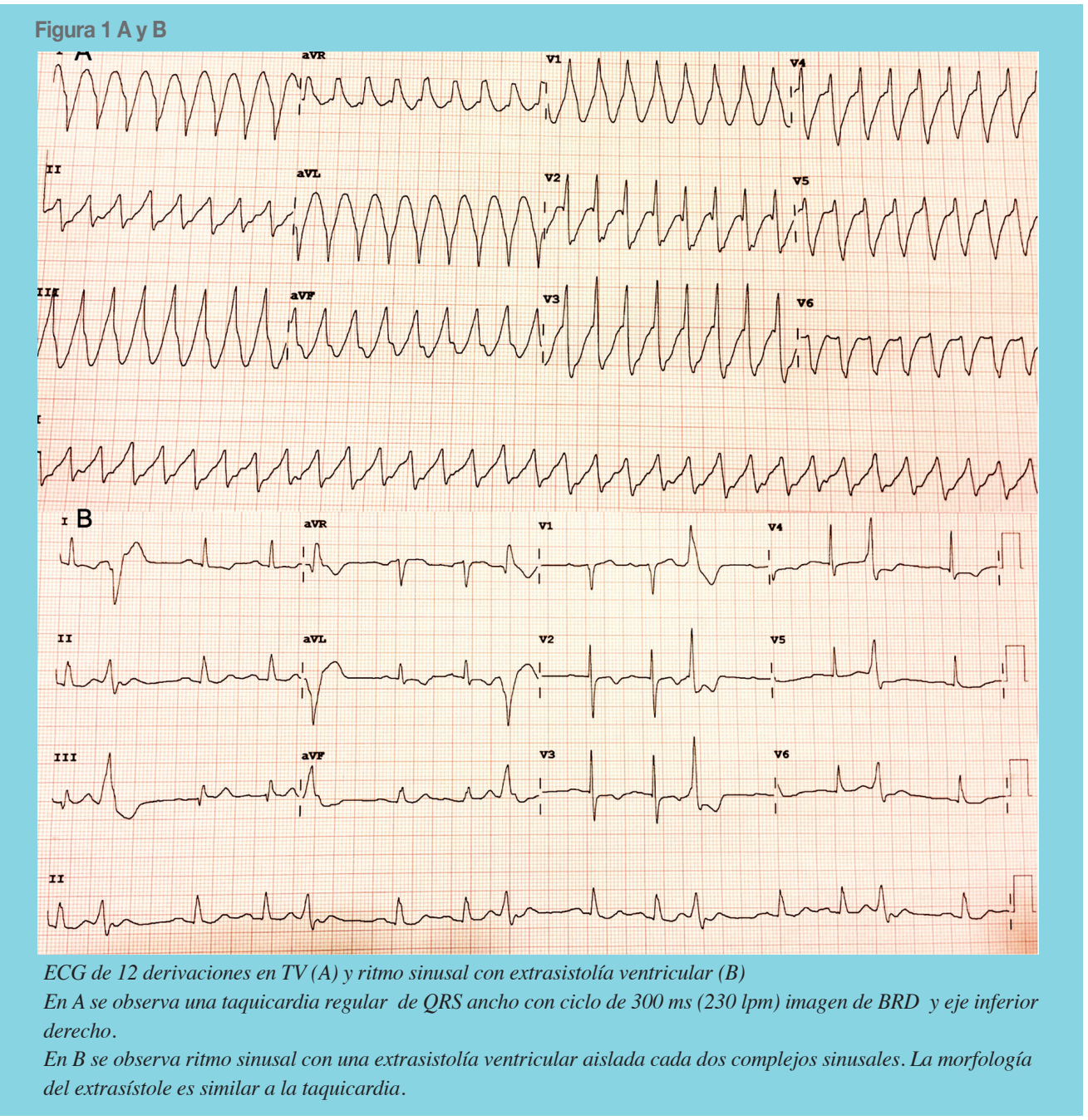

Tabla 1.

\begin{tabular}{|c|c|c|c|c|c|c|c|c|c|}
\hline Caso & Sexo & Edad & Cardiopatía & FEVI \% & CF & Presentación clínica & Arritmia & Antiarrítmicos & Intervenciones \\
\hline 1 & $\mathrm{H}$ & 55 & No & 56 & 1 & $\begin{array}{l}\text { Palpitaciones } \\
\text { Síncope }\end{array}$ & TV & $\begin{array}{l}\text { Atenolol } \\
\text { Amiodarona }\end{array}$ & Ablación (1) \\
\hline 2 & $\mathrm{H}$ & 74 & $\begin{array}{l}\text { Chagas } \\
\mathrm{MCl}\end{array}$ & 30 & 3 & $\begin{array}{l}\text { Sincope } \\
\text { Terapias DAI }\end{array}$ & TV & $\begin{array}{l}\text { Carvedilol } \\
\text { Amiodarona }\end{array}$ & $\begin{array}{c}\text { CRT - D } \\
\text { Ablación (2) } \\
\text { Bloqueo G. Estrellado }\end{array}$ \\
\hline 3 & $\mathrm{H}$ & 60 & MCDNI & 30 & 2 & $\begin{array}{l}\text { Sincope } \\
\text { Terapias DAI }\end{array}$ & TV & $\begin{array}{l}\text { Bisoprolol } \\
\text { Amiodarona }\end{array}$ & $\begin{array}{c}\text { DAI } \\
\text { Ablación (4) }\end{array}$ \\
\hline 4 & $\mathrm{H}$ & 60 & MCDNI & 30 & 3 & $\begin{array}{l}\text { MS recuperada } \\
\text { Terapias DAI }\end{array}$ & TV & $\begin{array}{l}\text { Carvedilol } \\
\text { Amiodarona }\end{array}$ & $\begin{array}{c}\text { CRT - D } \\
\text { Ablación (4) } \\
\text { Simpatectomía bilateral }\end{array}$ \\
\hline
\end{tabular}

$H=$ Hombre, $M C I=$ miocardiopatía isquémica, $M C D N I=$ miocardiopatía dilatada no isquémica, $F E V I=$ fracción de eyección del ventrículo izquierdo, $C F=$ clase funcional de la NYHA, DAI= desfibrilador automático implantable, $M S=$ muerte súbita, $T V=$ taquicardia ventricular, $C R T-D=$ desfibrilador con resincronizador. 
Figura 2 A y B

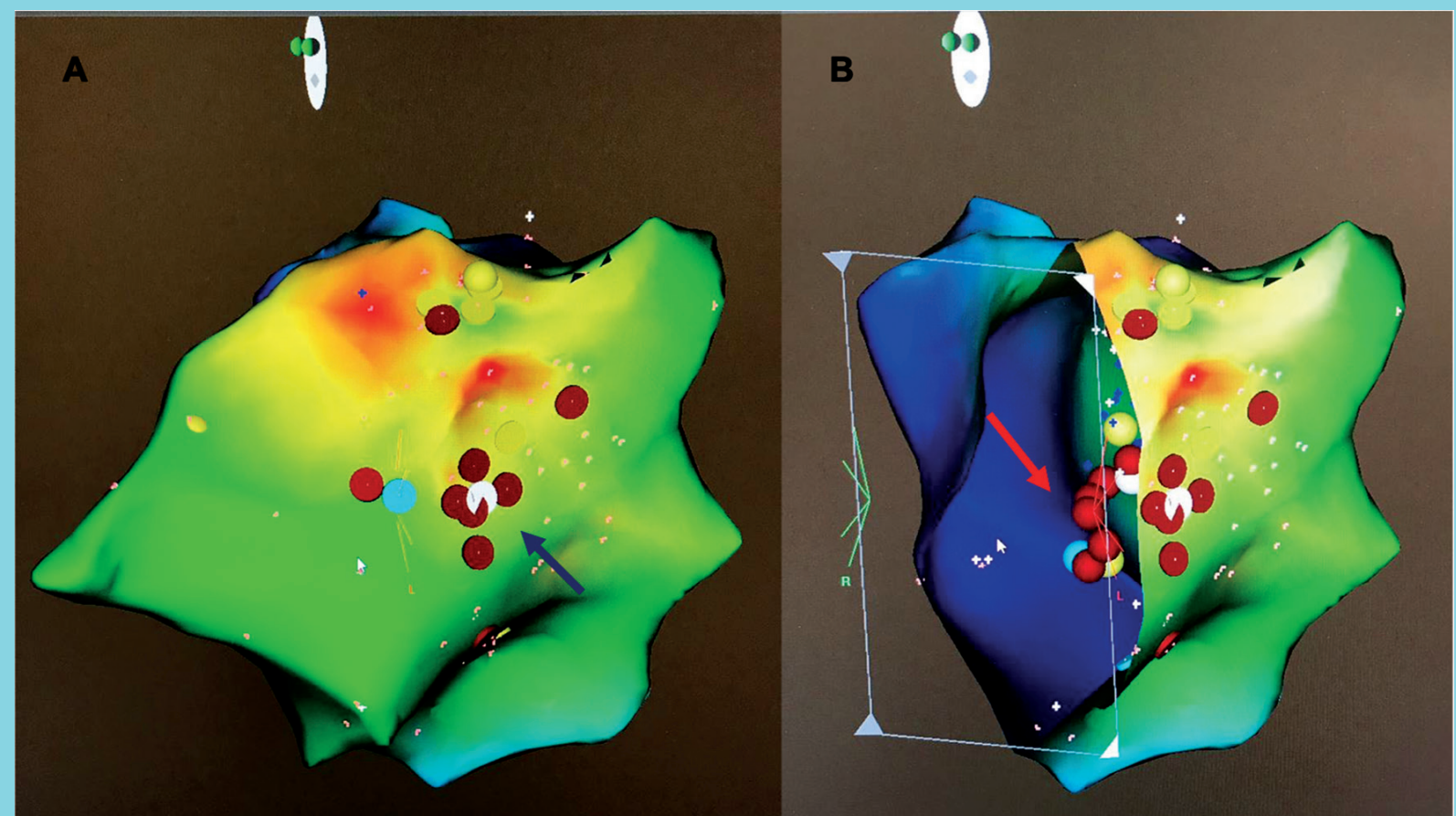

Mapa electroanatómico con sistema 3D CARTO de la pared lateral del ventrículo izquierdo (proyección lateral izquierda).

En A se observan las zonas de mayor precocidad obtenidas durante cartografía en TV (rojo). Destaca una zona amplia, lo que sugirió un origen distante a ésta. La flecha azul muestra las aplicaciones de RF sin resultado.

En B se observa la misma proyección que en A en un corte transversal del VI, indicando la zona de éxito de ablación (flecha roja) que da la impresión de estar "flotando" en el interior del VI, correspondiendo al músculo papilar anterolateral.

presencia de 3 o más episodios distintos de taquicardia ventricular (TV) y/o fibrilación ventricular (FV) durante un periodo de 24 horas y separados por más de 5 minutos entre cada episodio ${ }^{1}$. Se asocia a una alta morbimortalidad en pacientes con o sin un dispositivo de desfibrilación implantado (DAI). En este artículo se describen 4 casos clínicos de pacientes que se presentaron con una TA, se revisan sus causas y las alternativas terapéuticas (Tabla 1).

\section{Caso 1}

Varón de 55 años con hipertensión arterial. Ingresó a urgencias en mayo de 2019 por síncope. El electrocardiograma mostró una TV monomorfa sostenida (Fig 1a) a 230 lpm, que requirió cardioversión eléctrica (CVE). Presentó varios episodios de TV refractarias a betabloqueante y amiodarona tratadas con CVE. En ritmo sinusal destacó la presencia de una extrasistolía ventricular monomorfa frecuente con morfología similar a la TV. Se descartaron trastornos hidroelectrolítico, ácido base, metabólicos y tiroideos. El ecocardiograma mostró hipertrofia ventricular con función del ventrículo izquierdo (FEVI) normal. Coronariografía sin lesiones. La arritmia persistió después de sedación y conexión a ventilación mecánica. El Estudio electrofisiológico (EF) guiado por sistema de navegación CARTO (Biosense), demostró un origen focal anterolateral del VI (zona amplia de precocidad hasta $-20 \mathrm{~ms}$, ciclo de retorno $20 \mathrm{~ms}$ ). Aplicaciones de radiofrecuencia (ABL) (Contact Force, $40 \mathrm{~W} / 45^{\circ}$, irrigación $17 \mathrm{ml} / \mathrm{min}$ ) no interrumpieron la TV. Posicionamiento en músculo papilar anterolateral (MPAL) (guiado por resalte en el movimiento del catéter en radioscopía y al alejarse del apoyo mural en el sistema CARTO) (Fig 2) mostró una precocidad de - 35 ms. ABL interrumpió la TV y la volvió no inducible. PACEMAP en el MPAL con concordancia 12/12. Alta luego de 72 horas de monitorización, previo control ecocardiográfico que descartó insuficiencia mitral. No se implantó un desfibrilador. No repitió la arritmia en un seguimiento de 16 meses.

\section{Caso 2}

Varón de 74 años con miocardiopatía chagásica y coronaria, disfunción VI severa y BCRI. MP VVI en 2002 


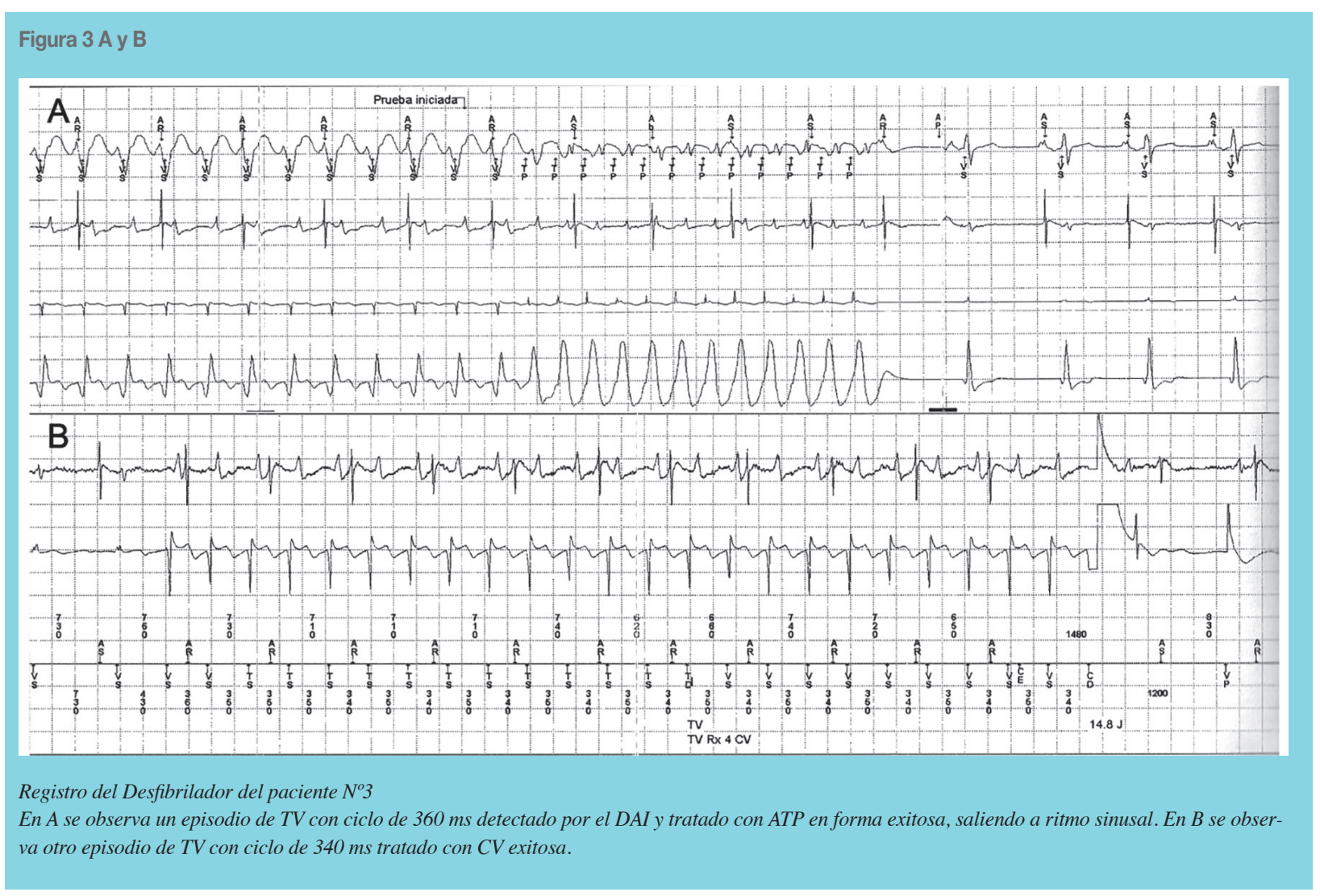

por síncope, cambio de generador e implante de nuevo electrodo el 2010 (se abandonó el electrodo disfuncional). El 2017 ingresó en urgencias por síncope y TVMS. La coronariografía mostró lesión severa de arteria circunfleja y se realizó una angioplastía con Stent no medicado, quedando en tratamiento antiarrítmico con amiodarona. Tres meses después se realizó un "upgrade" a Desfibrilador con resincronizador (CRT -D) por recurrencia de TV. En marzo 2019 ingresó a urgencias por episodios sincopales y descargas del desfibrilador en TA. La interrogación del dispositivo mostró seis episodios tratados con ATP y 4 con CV en las últimas 24 horas. Se descartaron causas isquémicas, metabólicas e hidroelectrolíticas. EF indujo dos TVMS. La primera TV con ciclo de $360 \mathrm{~ms}$. El abordaje endocárdico fue frustro, por lo que se realizó acceso epicárdico con ablación exitosa en pared lateral media de VI, con enlentecimiento del ciclo de la TV a 420 ms y luego interrupción. La segunda TV inducible, con morfología procedente del TSVD, fue imposible de abordar por adherencias intraventriculares derechas secundaria a los electrodos ${ }^{3}$ del VD.

En julio 2019 ingresó nuevamente por TA. Se realizó ABL endocárdica guiada por sustrato ( 2 morfologías de TV rápida, no mapeables). Se aumentó amiodarona a 400 mg al día y se optimizó el tratamiento de insuficiencia cardíaca.

En diciembre de 2019 ingresó por tercera vez en TA. Se desconectó el desfibrilador y se efectuó un bloqueo anestésico del ganglio estrellado izquierdo guiado por ecografía, con una reducción significativa de los episodios de TV, lo que sirvió de puente para aumentar la terapia farmacológica antiarrítmica y betabloqueante hasta el máximo tolerado por el paciente quien falleció 3 meses después durante otro episodio de tormenta arrítmica.

\section{Caso 3}

Varón de 60 años con miocardiopatía dilatada no isquémica, disfunción ventricular izquierda severa (FEVI $30 \%$ ) y deterioro leve de su CF (II, NYHA).

En 2010 se implantó un DAI bicameral por TVMS sincopal y se trató su insuficiencia cardíaca con vasodilatadores, betabloqueante y amiodarona.

En 2014 presentó descargas del DAI (8 episodios de TV tratados con ATP y CV en 24 horas). El EF indujo 2 morfologías de TV con ciclo de $240 \mathrm{~ms}$, mal toleradas por lo que se realizó CVE y luego una ablación con RF guiada por sustrato (zona de bajo voltaje basal posterolateral). Dos meses después repite TA por lo que se realizó un 
abordaje endo y epicárdico basado en sustrato por TV mal tolerada.

En 2019 nuevamente ingresó por TA. Se indujeron 3 morfologías de TV (1: $280 \mathrm{~ms}, 2: 280 \mathrm{~ms}, 3: 240 \mathrm{~ms}$ ), todas con imagen de BCRD, eje superior y morfología similar. Las dos primeras con estabilidad hemodinámica, que permitieron maniobras de sobreestimulación con ciclos de retorno idéntico al ciclo de la taquicardia, fusión oculta e interrupción durante RF a los 18 y 10 segundos respectivamente, quedando inducible la $\mathrm{TV}^{3}$ que no permitió estudio.

En 2020 ingresó a urgencias por dos descargas del DAI y mareo. Al momento de su ingreso se encontraba en TV a una frecuencia de $163 \mathrm{lpm}$, con buena tolerancia hemodinámica y sin terapia del DAI por encontrarse bajo el primer nivel de corte de detección. La iterrogación del dispositivo mostró 22 episodios de TV tratadas con ATP, dos episodios tratados con CV en las últimas 72 horas (Fig 3 A y B) y el episodio en curso de 4 horas de evolución con ciclo de 360 ms. Por primera vez en su evolución se logró obtener un ECG de 12 derivaciones durante un episodio de TV "clínica" con características muy particulares que orientaron al diagnóstico (Fig 4). Se administró en forma manual una terapia de sobreestimulación a $250 \mathrm{~ms}$ logrando conversión a ritmo sinusal.

El EF indujo TV clínica con igual longitud de ciclo y bien tolerada. Por las características específicas del ECG se realizó en forma dirigida un mapa de activación con sistema ENSITE Velocity de la región anterolateral del VI con especial énfasis en el MPAL, apoyado con imagen ecográfica, que confirmó la posición del catéter en la zona más apical de éste, se obtuvo una precocidad de - $40 \mathrm{~ms}$, un ciclo de retorno de $10 \mathrm{~ms}$ y fusión oculta (Fig 5). La ABL en el MPAL con $50 \mathrm{~W} / 50^{\circ}$ durante TV provocó un cambio sutil en la morfología del QRS (negativización en D3), enlentecimiento del ciclo y luego interrupción limpia de la TV (Fig 6). En esta oportunidad quedó sin arritmias inducibles con un protocolo agresivo de estimulación con tres extra-estímulos acoplados hasta su periodo refractario. Luego de 3 meses de seguimiento sin arritmias se suspendió la amiodarona, continuando la terapia con betabloqueante. En un seguimiento de 16 meses no ha presentado arritmias.

\section{Caso 4}

Varón de 60 años con miocardiopatía dilatada no is-

Figura 4.

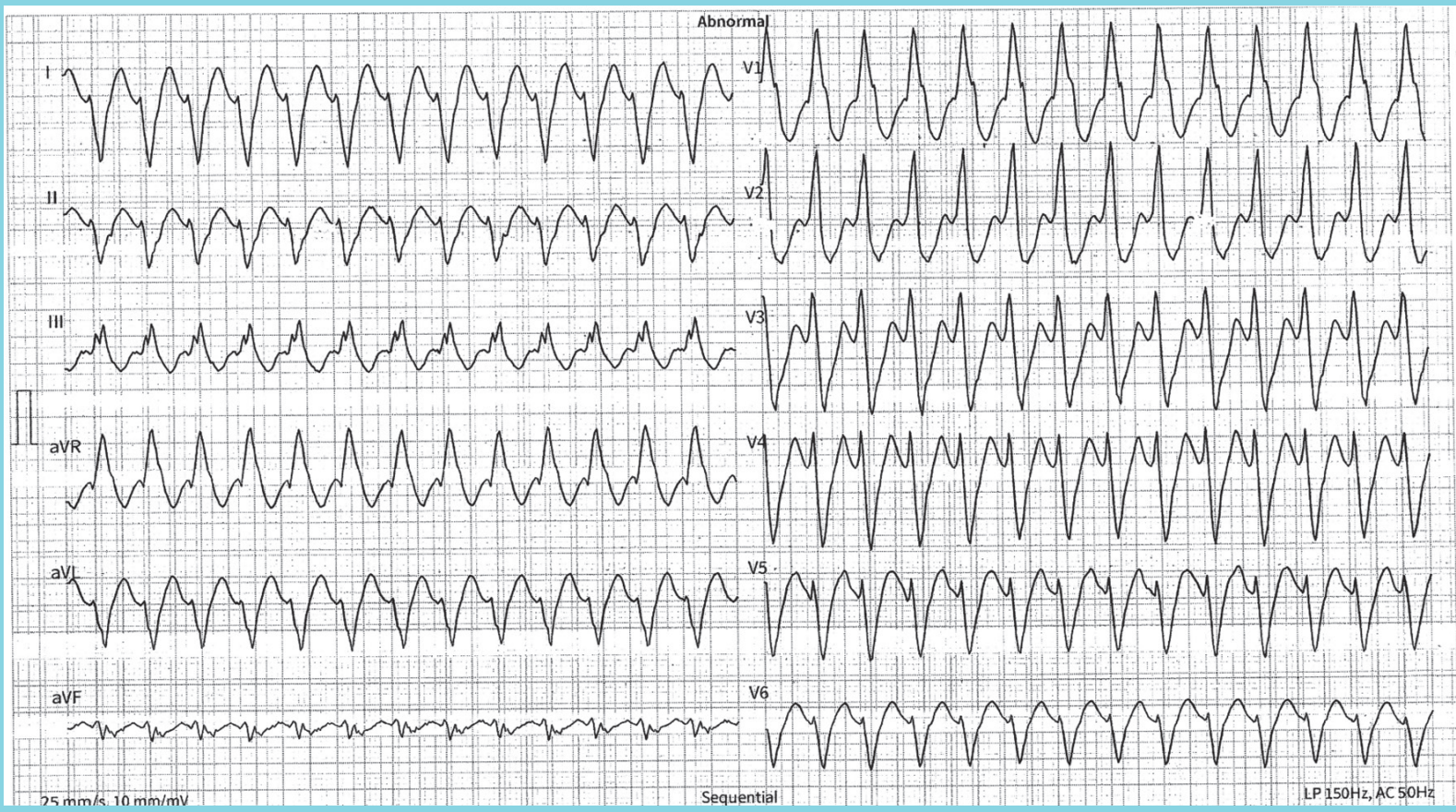

ECG de 12 derivaciones del paciente $N^{a}$ 3. Taquicardia regular a 180 lpm, de QRS ancho con morfología de BCRD y eje derecho. Destaca la discordancia de las derivaciones inferiores con QRS negativo en D2 y positivo en D3 (ver el texto) 


\section{Figura 5 A, B y C}
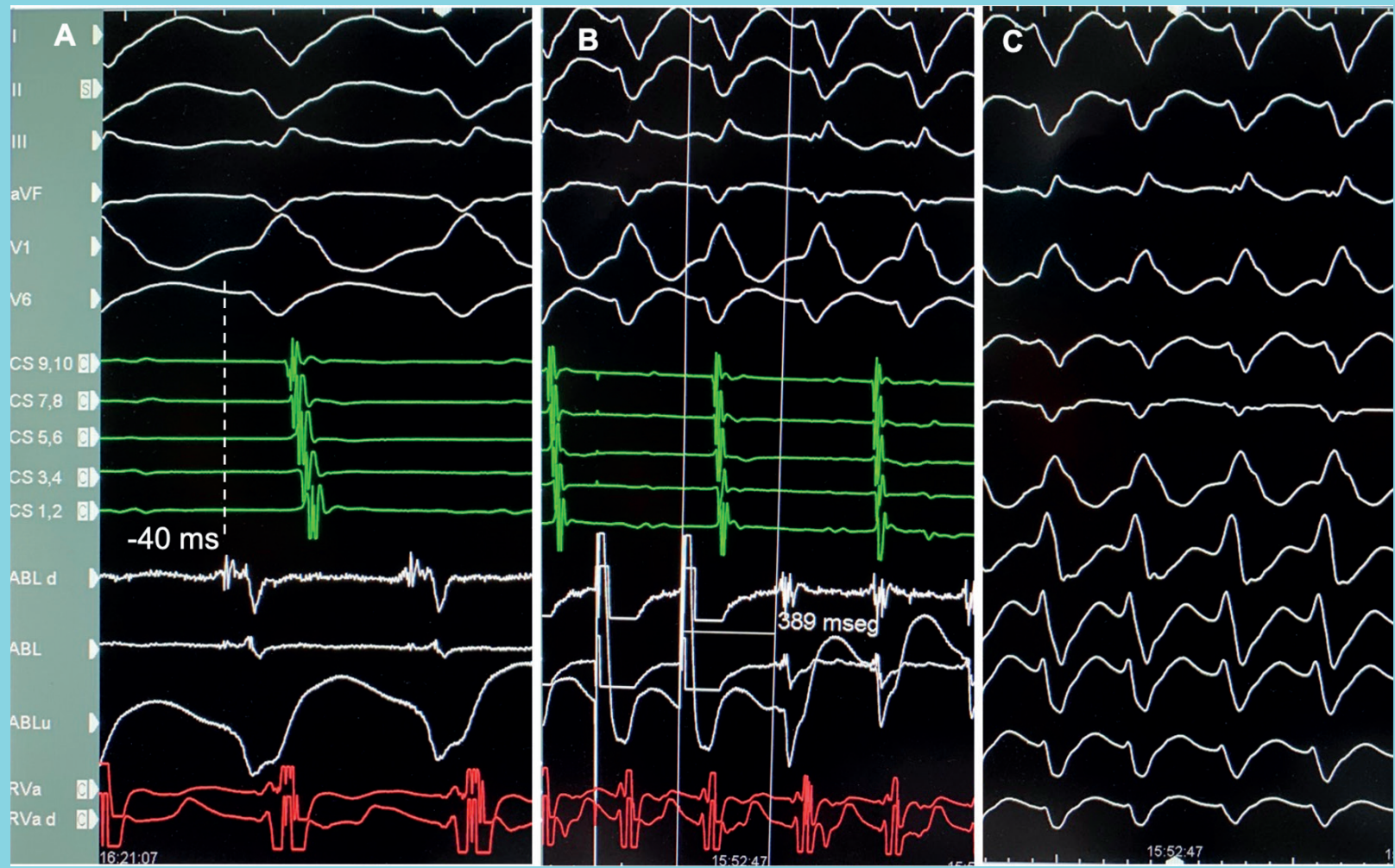

Registro ECG e intracavitario de la TV del paciente $N^{\circ} 3$

En A se muestran dos latidos de la TV $(100 \mathrm{~mm} / \mathrm{s})$. Los 6 primeros canales corresponden a derivaciones del ECG de superficie, los canales en verde al registro auricular de seno coronario seguido de tres canales de ablación (distal, proximal y unipolar). Los dos últimos canales registran la señal de VD. En el canal ablación distal se observa un potencial rápido presistólico que antecede al inicio del QRS en $40 \mathrm{~ms}$.

En B se observa misma distribución que en A a $50 \mathrm{~mm} / \mathrm{s}$. Estimulación desde el catéter de ablación 20 ms más rápido que la TV logra encarrilamiento de ésta, un intervalo post estimulación de $389 \mathrm{~ms}$ y un ciclo de retorno de $10 \mathrm{~ms}$.

En C se muestra el ECG de 12 derivaciones de los 2 últimos latidos encarrilados y los 2 primeros latidos luego del cese de la estimulación, observándose "fusión oculta".

quémica y disfunción ventricular severa (FEVI 30\%) y BCRI.

En 2013 se implantó un desfibrilador con resincronizador por muerte súbita recuperada. Tres meses después ingresó por descargas múltiples del DAI. La interrogación mostró 37 episodios de TV en las últimas 72 horas, con ciclo de 290 ms. La mayoría tratados con ATP y 2 de ellos con CV por ATP frustro. EF indujo TVMS con ciclo de 270 ms, imagen de BCRD, QRS negativo en D2 y aVF y positivo en D3 (discordancia de derivaciones inferiores, ILD) sugerente de origen en MPAL. Esta TV degeneró en FV por lo que requirió DF. El Mapa electro anatómico mostró una zona de bajo voltaje a nivel anterolateral del VI. Se realizó una ablación con RF del MPAL y luego basada en sustrato. La TV1 quedó no inducible, pero con protocolo agresivo se indujo una TV2 a $250 \mathrm{~ms}$ no tole- rada que se resolvió con CVE.

En 2018 ingresó por segunda TA. EF indujo una TV lenta con ciclo de 430 ms que permitió realizar maniobras de entrainment, obteniéndose buenos parámetros en región inferior - media del VI. La ABL sólo logró enlentecer el ciclo de la TV a $520 \mathrm{~ms}$, con interrupciones, pero reinducciones. La ABL epicárdica tuvo igual resultado (probablemente circuito intramural).

En 2019 ingresó por tercer episodio de TA. Se indujeron 4 morfologías de TV; dos de ellas con ciclo de 290 y $270 \mathrm{~ms}$ no toleradas termodinámicamente y 2 con ciclo de 420 y 390 ms que pudieron ser interrumpidas con RF en segmento medio de la pared lateral y en pared septal inferior del VI respectivamente.

En marzo 2021 ingresó nuevamente por el mismo diagnóstico. Se logró obtener registro electrocardiográfico de 
dos morfologías diferentes de TV: ínfero- septal y lateral de VI (Fig 7). En el EF apareció en forma espontánea la TV clínica con ciclo de $320 \mathrm{~ms}$, no sostenida. Fue imposible reproducirla con protocolos de estimulación ventricular, que sólo consiguieron inducir TV no clínica con ciclos inferiores a $290 \mathrm{~ms}$ y $\mathrm{FV}$ que requirieron CV/ DF. Se realizó homogenización de escara lateral e inferior identificando potenciales tardíos y medio diastólicos durante estimulación desde VD, sin éxito.

Tras un lapso de 72 horas de observación tras ablación, con amiodarona $300 \mathrm{mg}$ al día y metoprolol $100 \mathrm{mg}$ cada 12 horas, persistieron las arritmias ventriculares en for- ma incesante. Se observó que la estimulación sensorial incrementaba la ocurrencia de las arritmias. Teniendo en consideración esta observación, el historial arrítmico de este paciente y su respuesta parcial a fármacos y $\mathrm{ABL}$, se realizó una simpatectomía torácica bilateral (Fig 8), sin complicaciones, salvo hipotensión arterial leve que se manejó con adecuación de medicación. Luego de 72 horas de monitorización sin recurrencia de arritmias fue dado de alta A los dos meses se presentó con TV lenta (bajo nivel de corte del DAI), con tolerancia hemodinámica que permitió un mapeo y ablación exitosa en zona anterolateral media del VI.

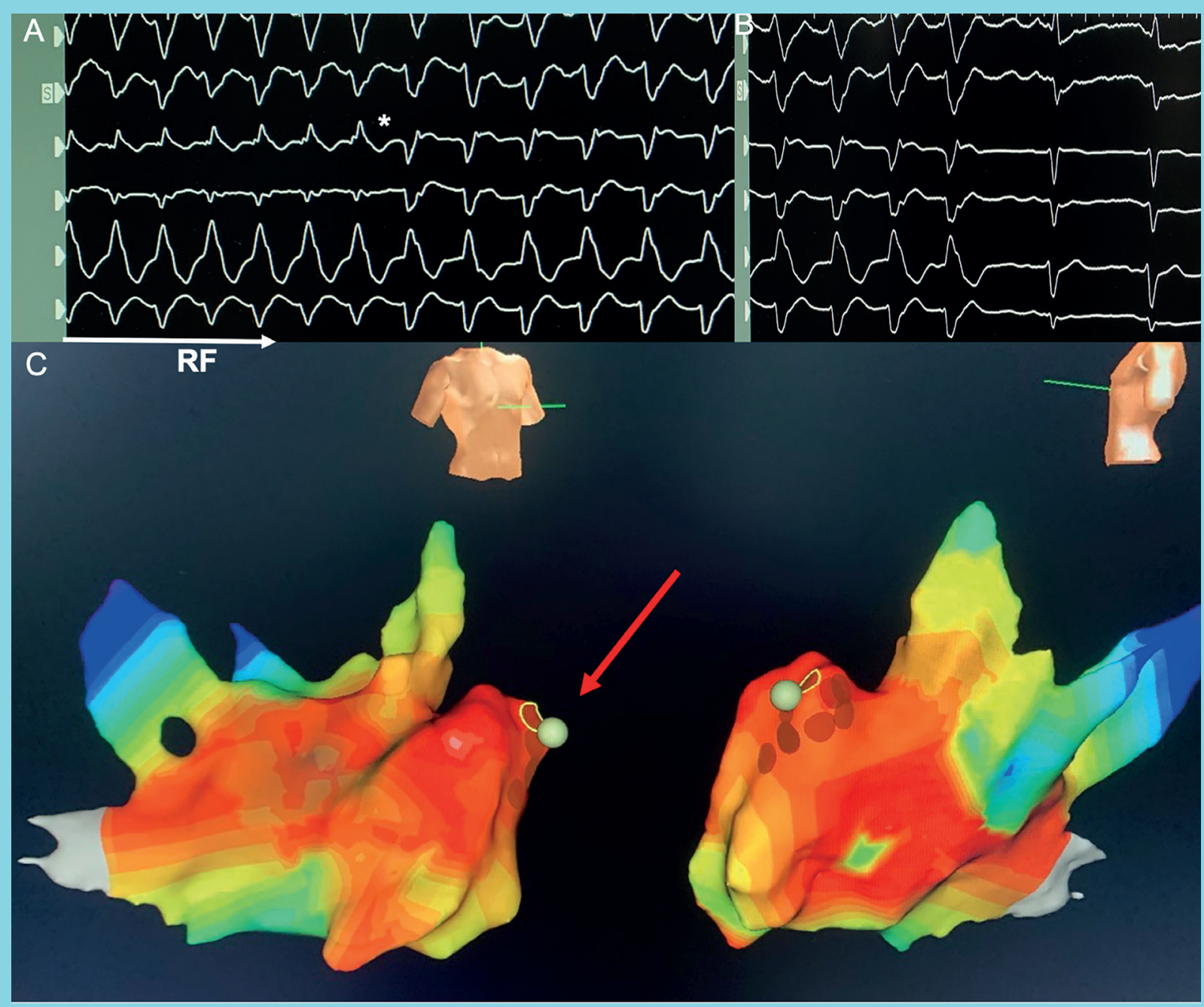

Ablación de la TV del paciente $N^{\circ} 3$

En A se muestra un registro ECG de 6 derivaciones durante el inicio de la ablación con RF en el MPAL. Se observa un cambio de morfología del QRS después del séptimo latido (*) indicativo de una modificación en el sitio de salida de la TV, enlentecimiento del ciclo y término de la taquicardia (B). En C se muestra el mapa de ENSITE del MPAL en proyecciones oblicuas derecha e izquierda. La flecha roja indica la zona de éxito. 


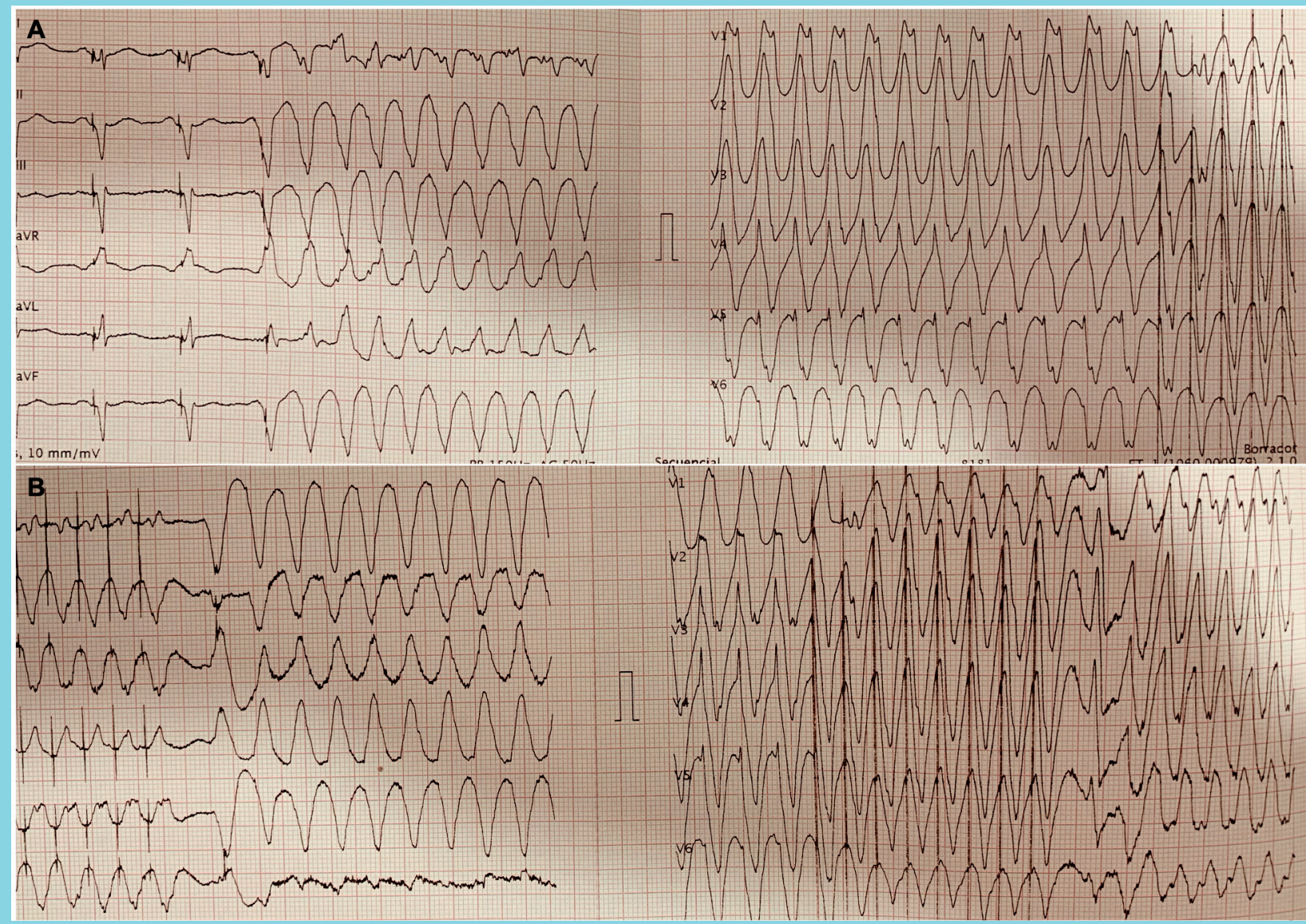

ECG de 12 derivaciones registrado en servicio de urgencia justo durante un episodio de TV del paciente $N^{\circ} 4$ En A, luego de 2 latidos estimulados por el resincronizador, se inicia una TVMS con imagen de BCRD y eje superior izquierdo. Se observa el inicio de una intervención del DAI (ATP). En B se observa la continuación de la terapia de ATP que generó una segunda morfología de TV con imagen de BCRI y eje $180^{\circ}$

\section{Discusión}

Presentamos 4 pacientes tratados por TA, en los cuales el manejo médico habitual no logró un control de los episodios de TV. En tres de ellos con múltiples terapias y en dos, incluso con terapias de excepción.

La incidencia de TA oscila entre un $10-20 \%$ en portadores de DAI por prevención secundaria y en torno al $4 \%$ en portadores de DAI por prevención primaria. El tiempo transcurrido desde el implante del DAI hasta el primer episodio de TA fue entre 5 y 18 meses $^{2}$.

La TA afecta, principalmente, a pacientes con miocardiopatía dilatada, sea de etiología isquémica o no. También puede afectar a pacientes sin cardiopatía estructural (Brugada - canalopatías), o incluso en pacientes con corazón sano, como el primer paciente de nuestra serie.

La TV monomórfica es la forma más frecuente de presentación de la TA. Se han descrito TV monomórficas (86-97\%), FV aislada (1-21\%), mixtos TV + FV (3-14\%) y TV polimórficas aisladas $(2-8 \%)^{3}$. De forma menos común se ha podido identificar la presencia de extrasistolía ventricular como "trigger" de la TA en dos situaciones: el infarto agudo de miocardio y pacientes sin cardiopatía estructural ${ }^{4}$.

La TA se asocia con un incremento de mortalidad de forma precoz, según los estudios en pacientes con DAI, tanto en prevención primaria como secundaria. En el MADIT II, constituyó el mayor predictor independiente de mortalidad cardíaca a medio plazo con un riesgo 


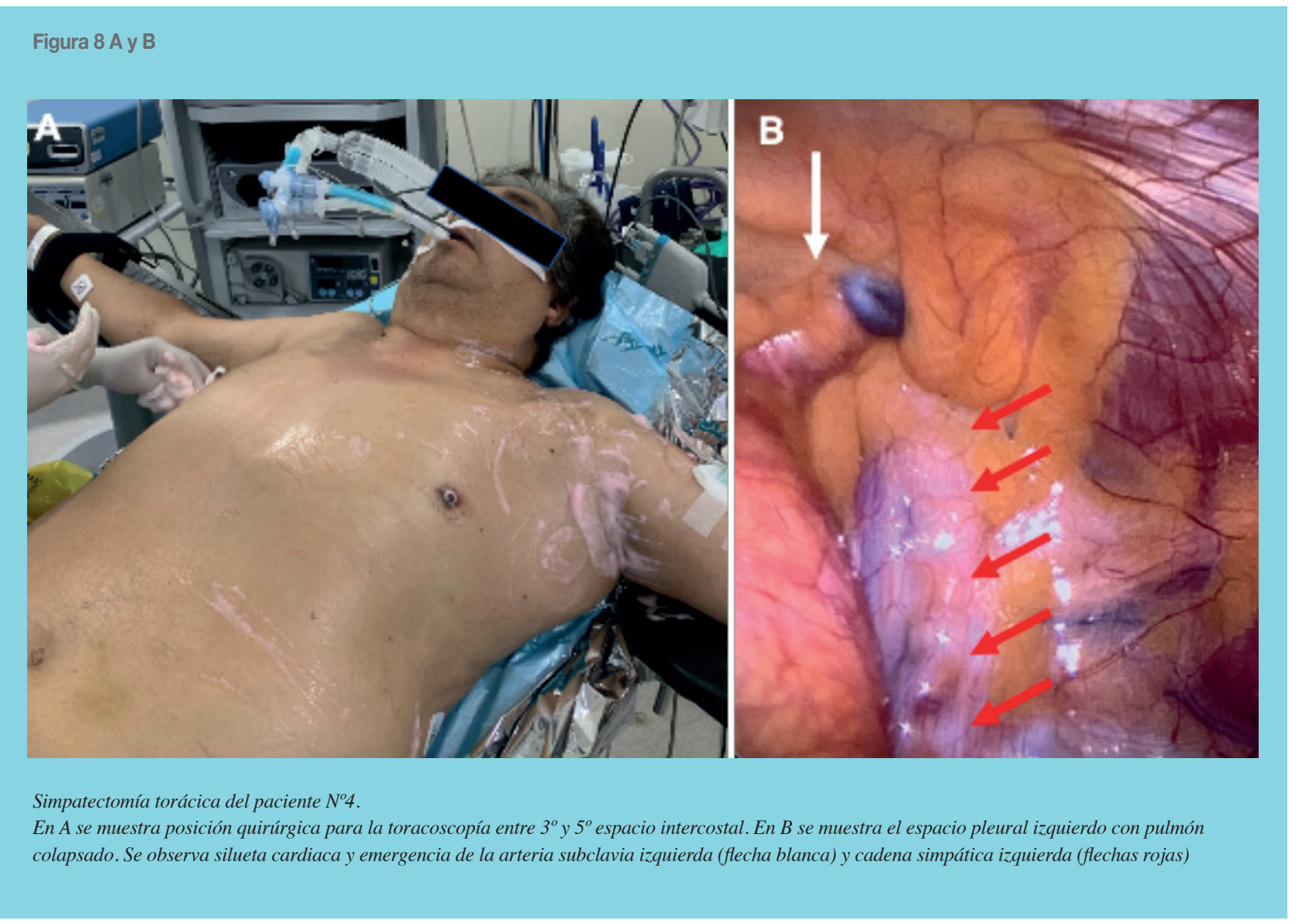

relativo (RR) de muerte 7 veces superior (y si se tienen en cuenta sólo los tres primeros meses tras el episodio, 18 veces superior), respecto a aquellos pacientes que no presentaron TV/ FV. En el SCD-HeFT, se demostró no sólo un aumento de mortalidad en pacientes con "choques" apropiados, sino también cuando los choques son inapropiados (RR de muerte de 5.6 veces en pacientes que presentaron choques apropiados y de 1.98 veces en pacientes con choques inapropiados respecto a los pacientes que no sufrieron choques $)^{5}$.

Los estudios de prevención secundaria mostraron lo mismo. Un metaanálisis que incluyó 5912 pacientes de 13 estudios (857 con TA), mostró que la TA es un potente factor asociado a mortalidad (RR: 3.15), trasplante cardíaco y hospitalización por insuficiencia cardíaca (RR: $3.39)^{6}$.

El aumento de la mortalidad, se debe al aumento de la concentración de calcio intracelular producido por los episodios repetidos de FV que conllevan a una reducción de la función ventricular izquierda. Los choques repetidos del DAI producen aturdimiento miocárdico, activación neuro hormonal y fibrosis 7 .
En la atención del paciente con TA, luego de su estabilización, es fundamental realizar un análisis sistemático que incluya la búsqueda y corrección de causas subyacentes o "triggers", ya sea de origen cardíaco, como por ejemplo la isquemia miocárdica, como de origen extracardíaco (desordenes metabólicos, hidroelectrolítico, equilibrio ácido - base y tiroideos). También es de utilidad conocer en el primer momento, cuales son las características basales del paciente, porque esto nos permitirá orientar mejor el conjunto de medidas que debemos ir adoptando en su manejo. Por ejemplo, si es un primer evento, entorno en el que ocurrió éste, si es portador de una cardiopatía conocida, que tipo de cardiopatía y si es portador de un dispositivo cardiaco. Las terapias del DAI, si bien permite terminar con los episodios de arritmias ventriculares en la mayoría de los casos y de esta forma prevenir la muerte súbita, no ayuda a la estabilización clínica. Al contrario, las terapias continuadas del DAI provocan un efecto hemodinámico desfavorable con estrés psicológico intenso, hiperactividad adrenérgica y disconfort del paciente ${ }^{1}$.

El tratamiento de los pacientes con TA se debe realizar 
en una unidad especializada que disponga de los recursos técnicos, farmacológicos y no farmacológicos necesarios para yugular la crisis, sin embargo, muchos pacientes pueden ingresar en centros de atención de salud primarios, no especializados o rurales que no dispongan de todos estos elementos.

En los pacientes portadores de dispositivos (DAI), es ideal contar con programadores y un equipo familiarizado con su uso con la finalidad de interrogarlo, determinar la carga arrítmica, el tipo de arritmia predominante involucrado, las terapias administradas y el tipo de estas (ATP/CV/DF). También permitirá identificar si las terapias fueron apropiadas o inapropiadas y/o apropiadas pero innecesarias. En este último punto es importante revisar la programación del dispositivo, determinar si se ajusta a las pautas habituales recomendadas, al historial de arritmias del paciente y su respuesta al tratamiento. Con el programador podemos también desactivar las detecciones / terapias del dispositivo, uno de los pilares en el manejo agudo de la TA para evitar choques múltiples (en caso de TV incesante), que en algunos casos pueden ser contraproducentes y agravar la condición clínica del paciente. En servicios clínicos que no dispongan de esta herramienta, pueden utilizar un imán para obtener el mismo efecto de detener los choques por parte del desfibrilador.

El manejo inicial de toda tormenta arrítmica debe incluir algunas acciones simultáneas y otras que se realizarán de forma escalonada, según la evolución clínica. En aquellos pacientes en los que identifiquemos un factor causal (ej.: isquemia miocárdica) o desencadenante sobre una condición crónica, debemos resolver esto lo antes posible, simultáneamente a las maniobras de RCP recomendadas ampliamente en guías clínicas.

\section{Terapia farmacológica}

Los fármacos de primera línea en el tratamiento de la TA son los betabloqueantes, siempre que no exista una contraindicación formal para su uso. Su utilización juega un papel esencial por su acción antiarrítmica y, fundamentalmente, por su efecto anti adrenérgico. Puede estar limitado su uso en presencia de una depresión severa de la función sistólica de ventrículo izquierdo o si existe inestabilidad hemodinámica. El tratamiento betabloqueante, especialmente si antagonizan los receptores tanto $\beta 1$ como $\beta 2$, ha mostrado un aumento en el umbral de FV y una reducción en la muerte súbita. En el estudio MADIT II, que incluyó pacientes con infarto de miocardio previo y $\mathrm{FEVI}<30 \%$, los pacientes en tratamiento con altas do- sis de betabloqueantes tuvieron una reducción del $52 \%$ en el riesgo relativo de muerte súbita en relación con los pacientes que no estaban en tratamiento betabloqueante ${ }^{8}$. La adición de tratamiento betabloqueante intravenoso en pacientes ya en tratamiento betabloqueante oral puede ayudar a controlar un episodio de TA.

La amiodarona se utiliza con frecuencia. Está contraindicada en el hipertiroidismo y en pacientes con QT prolongado. Dentro de los efectos adversos más frecuentemente observados con su utilización intravenosa está la hipotensión arterial y se debe tener precaución en pacientes con QT prolongado. De forma aguda, la amiodarona intravenosa bloquea los canales de $\mathrm{Na}+$, inhibe la recaptación de noradrenalina y bloquea los canales L-Ca+2, pero no actúa sobre el período refractario ventricular. De forma crónica, aumenta la refractariedad ventricular. El tratamiento con amiodarona se ha mostrado efectivo en prevenir recurrencias de episodios de TV/FV. El estudio OPTIC evaluó la eficacia del tratamiento con sotalol, betabloqueantes o amiodarona + betabloqueantes en la prevención de choques del DAI. Los pacientes en tratamiento con sotalol o amiodarona + betabloqueantes, presentaron una reducción de riesgo relativo del $56 \%$ de choques del DAI por episodios apropiados respecto a los pacientes en tratamiento betabloqueante aislado tras un período de seguimiento de 1 año 9 .

Otros fármacos antiarrítmicos como los de clase IA (Procainamida) o IC (Flecainida) pueden ser de utilidad en algunos casos seleccionados. El estudio randomizado PROCAMIO evaluó la eficacia y seguridad del tratamiento con procainamida i.v. vs amiodarona i.v en el tratamiento de 62 pacientes con taquicardia de QRS ancho tolerada. Procainamida fue más eficaz que amiodarona en la terminación de la TV (62\% vs 38\%) a los 40 minutos de la administración y presentó menor número de eventos cardiovasculares adversos ${ }^{10}$.

La lidocaína se ha utilizado por mucho tiempo con menor eficacia reportada versus amiodarona, sin embargo, en un estudio retrospectivo se ha mostrado útil en combinación con amiodarona en el tratamiento de la mayoría de las arritmias ventriculares refractarias ${ }^{11}$.

La sedación es también parte de la terapia aguda coadyuvante en la TA. En algunos casos será necesario llegar a un coma inducido farmacológico. El objetivo es disminuir la aferencia simpática nerviosa y humoral de catecolaminas. Está indicado en caso de TA refractaria a medidas iniciales, alta carga emocional y como puente mientras se resuelven alteraciones del medio interno o condicionantes agudos. 


\section{Ablación RF}

La ablación con catéter esta indicada en caso de arritmias ventriculares refractarias al tratamiento médico convencional y descartado causas agudas reversibles. Estudios han mostrado el beneficio en reducción de mortalidad del tratamiento precoz de ablación con catéter en pacientes con TA respecto al tratamiento con catéter de forma diferida, sólo cuando el tratamiento antiarrítmico fracasa ${ }^{12}$. La ablación ha mostrado reducir los choques del desfibrilador y la tormenta arrítmica en comparación a la estrategia de terapia farmacológica. Puede ser utilizada tanto en la "prevención" como en el "tratamiento" de la TA. En "prevención" existen dos estudios randomizados que han evaluado la ablación de TV precoz tras implante de DAI por prevención secundaria en pacientes con infarto de miocardio previo. En el primer estudio, Reddy et al. incluyeron 128 pacientes con TV sin tratamiento antiarrítmico y los randomizaron a ablación de sustrato de TV vs tratamiento convencional. Tras 22.5 meses de seguimiento, los pacientes sometidos a ablación tuvieron menos choques por TV/ FV del DAI (9\% vs $31 \%$ grupo control) y menos TV (12\% vs $33 \%$ grupo control $)^{13}$. En el segundo estudio, Kuck et al. incluyeron 110 pacientes randomizados a ablación con catéter vs tratamiento antiarrítmico. El 35\% de pacientes estaban basalmente con amiodarona. El número de episodios arrítmicos tratados por el DAI por paciente y por año fue significativamente más bajo en el grupo de ablación ( 0.2 vs. 3.0 en el grupo control) ${ }^{14}$.

Carbucicchio et al. publicaron un estudio prospectivo para evaluar el resultado a corto y largo plazo de la ablación con catéter en pacientes con TA. Después de hasta 3 procedimientos, el $86 \%$ de pacientes no tuvieron ninguna arritmia ventricular inducible en el estudio electrofisiológico, el $92 \%$ estuvieron libres de nuevos episodios de TA y el 66\% libres de cualquier TV tras 22 meses de seguimiento $^{15}$.

En un meta-análisis de 471 pacientes con TA, la tasa de éxito inicial del procedimiento de ablación de todas las TV fue del $72 \%$, la mortalidad del $0.6 \%$ y la tasa de recurrencias del $6 \% 16$.

La evidencia en pacientes con miocardiopatía no isquémica es menor y sólo existen reportes de series con pocos pacientes en algunos subgrupos de miocardiopatías específicas ${ }^{17,18}$.

Recientemente, se ha publicado una serie que compara los resultados de la ablación con catéter en pacientes con TA de origen isquémico y no isquémico. Lo más relevante, es que no se detectaron diferencias en el resultado de la ablación entre los pacientes isquémicos con o sin TA, mientras que en los pacientes no isquémicos, los pacientes con TA tuvieron mayor incidencia de recurrencia de arritmias ventriculares y de muerte/ trasplante que los pacientes sin $\mathrm{TA}^{19}$.

El mayor rendimiento se logrará en paciente con TV, aunque existen reportes en la literatura que muestran éxito en pacientes con fibrilación ventricular desencadenada por extrasístoles "malignos" y en tormentas arrítmicas asociadas al síndrome de Brugada ${ }^{4,20}$.

Las técnicas de ablación son variadas, pero en términos generales, existen dos grupos; aquellas basadas en la inducción y cartografía clásica (sobreestimulación durante taquicardia para identificar el componente crítico del circuito) y aquellas basadas en la ablación anatómica empírica del sustrato. En nuestra serie realizamos una mezcla de ambas; en los casos con arritmias inducibles, sostenidas y con cierta estabilidad hemodinámica, siempre optamos por un abordaje clásico ("mapeo en taquicardia") y en aquellos casos sin inducibilidad o con TV rápidas mal toleradas o sincopales, una ablación por sustrato. Para tal efecto, es indispensable contar con algún sistema de mapeo electro anatómico.

En pacientes graves o con inestabilidad hemodinámica que impida el mapeo de estas arritmias, se puede realizar una ablación apoyada con algún dispositivo de asistencia circulatoria.

Últimamente, se ha introducido el concepto, al igual que en la ablación de fibrilación auricular, de cuantificar la reducción de la carga arrítmica tras ablación de taquicardia ventricular, en lugar de considerar la recurrencia como una variable dicotómica, pues dicha reducción de la carga conlleva beneficios clínicos derivados de menos choques del DAI, menos hospitalizaciones, etc. Quinto et al, en una cohorte de 169 pacientes con cardiopatía estructural y TV monomorfa sostenida, realizaron una técnica de ablación de sustrato, con eliminación de los canales y todos los potenciales aislados encontrados y la no inducibilidad final, como objetivos del procedimiento. La carga arrítmica previa se cuantificó en todos los casos previo y después del procedimiento. Se consiguió la no inducibilidad completa tras la ablación en el $71 \%$ de los casos, con ausencia de potenciales tardíos en el $66 \%$. Tras un seguimiento medio de 3,14 años, la tasa de recurrencia global fue del 41,4\%, sin diferencias según tipo de cardiopatía. Se observó una significativa reducción de la carga arrítmica tras la ablación, del 99,6\% (21,8 episodios anuales de media preablación vs. 1,8 episodios postablación, $\mathrm{p}<0,001)$. Comparando la carga arrítmica pre 
y postablación exclusivamente de aquellos con al menos una recurrencia de taquicardia ventricular, la reducción de la carga fue del $69 \%$, e igualmente se constataron reducciones significativas de choques apropiados y de episodios de tormenta arrítmica. La eliminación incompleta de potenciales tardíos aislados y la FEVI, fueron variables predictoras independientes de recurrencia ${ }^{21}$.

El éxito de esta terapia a largo plazo depende de factores técnicos (el circuito debe ser relativamente constante y anatómicamente asequible), como de factores que pueden ir modificándose en el tiempo por la progresión de la enfermedad de base. Uno de los aspectos fundamentales, que incide en el éxito de la ablación, es poder disponer de un ECG de 12 derivaciones de la TV (llamada TV clínica). Aunque parece un requerimiento muy simple, muchas veces no es posible disponer de él por diversas razones: el paciente llega a un servicio de urgencias muy inestable y sólo se conecta a un monitor electrocardiográfico o simplemente no hay cultura de hacerlo o, en prácticamente la totalidad de pacientes portadores de un desfibrilador que ingresan por terapias múltiples, no logramos obtener un ECG durante taquicardia. El ECG de 12 derivaciones en TV nos aporta mucha información: 1 . El ciclo de la TV clínica, junto con su comportamiento hemodinámico, nos ayudará a planificar la estrategia de ablación; 2. La morfología del QRS nos orientará hacia tipos específicos de TV y su localización; 3. Poder corroborar si las arritmias inducidas en el laboratorio corresponden con la TV clínica del paciente. En 3 pacientes de nuestra serie, pudimos tener en al menos algunos de los episodios de TA, un ECG de 12 derivaciones (pacientes 1,3 y 4) y en dos de ellos ( 1 y 3 ) consideramos que incidió directamente en el resultado del procedimiento. En el paciente número 3 , habiendo tenido 3 ablaciones previas con resultados parciales tal como se describió, en el cuarto procedimiento ingresó a urgencias en TV por debajo del nivel de corte de la primera zona de detección de TV del desfibrilador, lo que permitió realizar un ECG de 12 derivaciones que mostró una TV izquierda con un eje superior izquierdo y una discordancia de las derivaciones inferiores (QRS - D2 y + D3), lo que orientó el diagnóstico de TV del músculo papilar anterolateral (MPAL). Se ha descrito en la literatura que la discordancia de las derivaciones inferiores (ILD, inferior lead discordance) es un patrón característico de algunas TV de localización específicas. Enríquez et al, describió una serie de 281 pacientes ingresados para una ablación por arritmia ventricular idiopática, en la cual 25 de ellos $(8,9 \%)$ presentaron el patrón electrocardiográfico de ILD. La localización final del foco arrítmico se realizó con mapeo electro anatómico y ecografía intracardíaca. En pacientes con un patrón positivo/negativo (D2/D3) $(n=18)$, el foco estuvo en la región parahisiana en 14 casos o en la banda moderadora o papilares del ventrículo derecho en 4 casos. En pacientes con patrón negativo/positivo (D2/D3) (n=7), todos los focos fueron localizados en el MPAL ${ }^{22}$.

\section{Modulación simpática}

El sistema nervioso autónomo juega un rol crítico en la iniciación y mantención de las arritmias ventriculares y éstas por si mismas pueden modular el efecto simpático sobre el corazón. Estudios en animales han demostrado que la isquemia miocárdica puede provocar un remodelado del ganglio estrellado e incrementar la actividad simpática. En estudios de medicina nuclear utilizando un análogo de la norepinefrina (I-meta-iodobenzylguanidina: I-MIBG) se puede visualizar la inervación simpática cardíaca y se ha encontrado anormalmente aumentada en pacientes con cardiopatía isquémica y no isquémica.

En pacientes con TA, la modulación simpática está indicada siempre y desde el inicio del manejo de estos pacientes. Existen varios niveles de modulación simpática; desde la sedación/coma farmacológico y el uso de fármacos convencionales betabloqueantes a intervenciones más radicales como el bloqueo anestésico del ganglio estrellado (GE), la anestesia epidural torácica, la ablación con radiofrecuencia del GE, la simpatectomía torácica uni o bilateral y más recientemente la denervación simpática renal.

El ganglio estrellado o ganglio cervicotorácico está presente en el $80 \%$ de la población por la fusión del ganglio cervical inferior y el primer ganglio simpático torácico, encontrándose en frente del cuello de la primera costilla y extendiéndose hasta la unión de la séptima vértebra cervical y la primera torácica. De su porción superior emergen fibras simpáticas oculares y faciales, mientras que de su porción inferior, fibras simpáticas cardíacas. En el área cardiovascular, el bloqueo del GE está indicado en la TA que no responde a medidas convencionales. Es una medida transitoria útil mientras se resuelve la causa que está originando la TA (ej: isquemia miocárdica). Se puede realizar con uno o dos anestésicos locales de vida media corta y larga (lidocaína y/o bupivacaina) por técnica guiada por referencias anatómicas, radiológicas o ecográficas, que es la usada actualmente. Requiere estar en conocimiento anatómico de las estructuras de la zona baja del cuello. Su eficacia es evaluada por la disminución o cese de la carga arrítmica y por la 
aparición de una complicación, en este caso transitoria, el síndrome de Horner: miosis, ptosis palpebral y anhidrosis ipsilateral. Meng et al, en una revisión sobre la eficacia del bloqueo del GE en la TA en 23 estudios (38 pacientes, FEVI media de $31 \%, 15$ pacientes con IAM y 7 pacientes con QT prolongado) mostraron una reducción significativa de la carga de arritmias ventriculares (12,4 vs 1.04 episodios 7día, p<0.001) y una reducción del número de choques externos y administrados por el DAI (10 vs 0.05 choques /día, $\mathrm{p}<0.01)^{23}$. Tian et al, en un estudio que incluyó 30 pacientes consecutivos con TA refractaria a fármacos, tratados con bloqueo del GE (15 pacientes GE izquierdo y 15 pacientes bilateral), mostraron que, a las 24 horas, $60 \%$ de los pacientes estaban libres de arritmias ventriculares. La mortalidad fue significativamente menor en aquellos que controlaban vs los que continuaban con arritmias (5,6 vs 50\%, $\mathrm{p}=0.009)$. La interrogación del DAI de estos pacientes mostró una reducción significativa del $92 \%$ en los episodios de arritmias ventriculares desde 26 a 2 episodios en las 72 horas posteriores al bloqueo del GE $(p<0.001) 24$. En nuestra serie, el paciente $\mathrm{n}^{\circ} 2$ portador de una miocardiopatía mixta chagásica e isquémica, tuvo una respuesta satisfactoria al bloqueo del GE izquierdo realizado con técnica ecográfica, con aparición de síndrome de Horner transitorio y cese parcial de la TA durante las primeras 6 horas del procedimiento.

La denervación simpática cardíaca (DSC) mediante simpatectomía torácica ha surgido como un tratamiento complementario para pacientes con TA o arritmias ventriculares refractarias a tratamiento ${ }^{25}$. Es una técnica utilizada para tratar la hiperhidrosis refractaria a tratamiento convencional. Su uso en arritmias se evaluó inicialmente en pacientes con taquicardia polimórfica catecolaminérgica y en síndrome de QT $\operatorname{largo}{ }^{26}$, pero también se ha demostrado que la DSC reduce la carga de arritmias ventriculares en pacientes con cardiopatía estructural subyacente. Hoy en día se realiza mediante toracoscopía con incisiones en la línea media axilar en el quinto y tercer espacio intercostal ${ }^{27}$. Utilizando un electrobisturí se separa y extirpa la cadena ganglionar desde la quinta hasta el GE, teniendo la precaución de extirpar sólo el tercio inferior de éste para evitar el Sd de Horner. Las experiencias iniciales realizaban una simpatectomía izquierda, pero las series actuales reportan la simpatectomía bilateral como abordaje más frecuentemente utilizado. Bourke et al estudiaron en una serie de 14 pacientes con TV de los cuales 12 tenían TA, el efecto de la anestesia torácica epidural y la denervación simpática cardíaca izquierda.
Ambas técnicas se asociaron con una reducción significativa en la carga arrítmica de los pacientes ${ }^{28}$. En casos de fracaso de la denervación simpática izquierda, se puede realizar una denervación simpática derecha con mejoría de los resultados. Se ha publicado un beneficio de la denervación simpática cardíaca bilateral con un $48 \%$ de pacientes libres de choques por el desfibrilador vs $30 \%$ en los pacientes solamente con denervación simpática cardíaca izquierda y una reducción en el número de choques en el $90 \%$ de los pacientes al cabo de 1 año de seguimiento 29 . Richardson et al, reportaron una serie de 7 pacientes dentro de 250 pacientes referidos a ablación de arritmias ventriculares en un plazo de 4 años. Sólo en el primer caso realizaron una simpatectomía izquierda, en el resto bilateral. Todos los pacientes habían fracasado a terapias farmacológicas con hasta tres fármacos y hasta 2 intentos de ablación previa. Luego de la DSC, ningún paciente tuvo recurrencia de arritmias ventriculares en el seguimiento de 7 meses $^{30}$. En nuestra serie, se realizó con éxito y sin complicaciones una simpatectomía bilateral en el paciente $\mathrm{N}^{\circ} 4$, luego de agotar terapias farmacológicas y de ablación RF con resultados parciales, con necesidad de reablación de una TV lenta dos meses después.

La denervación renal (DNR) se ha descrito como un tratamiento neuromodulador adicional para arritmias ventriculares recurrentes, TA y muerte súbita ${ }^{31}$. Los estudios han demostrado que la DNR por catéter es un tratamiento eficaz en pacientes con hipertensión resistente, con un excelente perfil de seguridad ${ }^{32}$. Inicialmente los resultados fueron dispares, pero un mejor entendimiento de la anatomía nerviosa simpática peri renal ha permitido modificar la técnica y desarrollar mejor tecnología, con mejores resultados en los estudios clínicos actuales (SPYRAL HTN -ON -OFF MED) ${ }^{33,34}$. La DNR se está evaluando actualmente como una terapia adyuvante en un espectro de enfermedades cardiovasculares moduladas simpáticamente, que incluyen el metabolismo alterado de la glucosa, hipertrofia ventricular izquierda (HVI) y disfunción diastólica, insuficiencia cardíaca congestiva, apnea obstructiva del sueño, y fibrilación auricular ${ }^{35}$, 36. Mediante un acceso endovascular arterial renal con un catéter específico de RF, se generan lesiones circunferenciales en forma de espiral para destruir las fibras simpáticas ubicadas en la grasa peri renal, principalmente cercano a la bifurcación arterial renal. El procedimiento produce una modulación simpáticas eferentes y aferentes alrededor de la pared de las arterias renales y una disminución de las catecolaminas circulantes.

Los efectos beneficiosos de la DNR en la reducción de 
las arritmias cardíacas en pacientes hipertensos y no hipertensos sugieren un mecanismo común asociado con la reducción de la noradrenalina. Esto puede resultar en reducciones de la frecuencia cardíaca en reposo, ectopias ventriculares y a un tono vagal mejorado o efectos simpáticos reducidos 37,38 .

La DNR fue eficaz para reducir la carga arrítmica y abolir la tormenta eléctrica en pacientes con cardiopatía estructural y arritmias ventriculares refractarias a fármacos, incluyendo aquellos con fracaso previo de ablación.

Una revisión de 7 artículos (121 pacientes, FEVI 30\%, ablación previa 46\%) mostró una reducción significativa de las terapias del DAI, con una diferencia media estandarizada (SMD) de -3.11 ( $<<0.001)$, reducción del número de arritmias ventriculares refractarias (SDM -2.82; $\mathrm{p}<0.001$ ), reducción de ATP (SMD -2.82; $\mathrm{p}=0.002$ ) y reducción de choques apropiados (SMD -2.82; $\mathrm{p}=0.002)^{39}$. La DNR se constituye como opción de tratamiento de segunda línea en pacientes con TA y FEVI reducida cuando la ablación con catéter convencional y múltiples intentos de tratamiento médico no logran controlar las arritmias ventriculares.

\section{Referencias:}

1. CARSTEN I, SERGE B. Electrical storm in patients with an implanted defibrillator: a matter of definition. Ann Noninvasive Electrocardiol. 2007; 12:375-82.

2. SESSELBERG HW, HUANG DT, ZAREBA W, ANDREWS M, MCNITT S, MCCLINITIC B et al. Storms of ventricular tachycardia/fibrillation in MADIT II patients. Heart Rhythm 2005; 2: S205.

3. HOHNLOSER SH, AL-KHALIDI HR, PRATT CM, BRUM JM, TATLA DS, TCHOU P et al. Electrical storm in patients with an implantable defibrillator: incidence, features, and preventive therapy: insights from a randomized trial. Eur Heart J 2006; 27: 3027-3032.

4. HAÏSSAGUERRE M, SHODA M, JAÏS P, NOGAMI A, SHAH DC, KAUTZNER J et al. Mapping and ablation of idiopathic ventricular fibrillation. Circulation 2002; 106: 962-967.

5. POOLE JE, JOHNSON GW, HELLKAMP AS, ANDERSON J, CALLANS DJ, RAITT MH et al. Prognostic importance of defibrillator shocks in patients with heart failure. N Engl J Med 2008; 359: 1009-1017.

6. GUERRA F, SHKOZA M, SCAPPINI L, FLORI M, CAPUCCI A. Role of electrical storm as a mortality and morbidity risk factor and its clinical predictors: a meta-analysis. Euro- pace. $2014 ; 16: 347-53$

7. RUNSIÖ M, BERGFELDT L, BRODIN LA, RIBEIRO A, SAMUELSSON S, ROSENQVIST M. Left ventricular function after repeated episodes of ventricular fibrillation and defibrillation assessed by transoesophageal echocardiography. Eur. Heart J. 1997;18:124-31.

8. MOSS A, ZAREBA W, HALL WJ, KLEIN H, WILBER D, CANNOM D et al. Prophylactic implantation of a defibrillator in patients with myocardial infarction and reduced ejection fraction. N. Engl. J. Med. 2002;346:877-83

9. CONNOLLY S, DORIAN P, ROBERTS R, GENT M, BAILIN S, FAIN E et al. Comparison of beta-blockers, amiodarone plus beta-blockers, or sotalol for prevention of shocks from implantable cardioverter defibrillators: the OPTIC Study: a randomized trial. JAMA. 2006;295:165-71.

10. ORTIZ M, MARTÍN A, ARRIBAS F, COLL-VINENT B, DEL ARCO C, PEINADO R, ALMENDRAL J. Randomized comparison of intravenous procainamide vs. intravenous amiodarone for the acute treatment of tolerated wide QRS tachycardia: the PROCAMIO study. Eur Heart J.2017 May 1;38:1329-1335.

11. YOSHIE K, TOMITA T, TAKEUCHI T, OKADA A, MIURA 
T, MOTOKI H, IKEDA U. Renewed impact of lidocaineon refractory ventriculararrhythmias in the amiodarone era. Int J Cardiol. 2014 Oct 20; 176: 936-40.

12. FRANKEL D, MOUNTANTONAKIS S, ROBINSON M, ZADO E, CALLANS D, MARCHLINSKI F. Ventricular tachycardia ablation remains treatment of last resort in structural heart disease: argument for earlier intervention. J. Cardiovasc. Electrophysiol. 2011; 22:1123-8.

13. Reddy V, Reynolds M, Neuzil P, Richardson A, Taborsky M, Jongnarangsin $\mathrm{K}$ et al. Prophylactic catheter ablation for the prevention of defibrillator therapy. N. Engl. J. Med. 2007; 357:2657-65.

14. KUCK KH, SCHAUMANN A, ECKARDT L, WILLEMS S, VENTURA R, DELACRÉTAZ E et al. Catheter ablation of stable ventricular tachycardia before defibrillator implantation in patients with coronary heart disease (VTACH): a multicentre randomised controlled trial. Lancet. 2010;375:31-40.

15. CARBUCICCHIO C, SANTAMARIA M, TREVISI N, MACCABELLI G, GIRALDI F, FASSINI G et al. Catheter ablation for the treatment of electrical storm in patients with implantable cardioverter-defibrillators: short and long-term outcomes in a prospective single-center study. Circulation 2008.

16. NAYYAR S, GANESAN A, BROOKS A, SULLIVAN T, ROBERTS-THOMSON K, SANDERS P. Venturing into ventricular arrhythmia storm: a systematic review and meta-analysis. Eur. Heart J. 2013;34:560-71.

17. MERINO JL, PEINADO R, FERNANDEZ-LOZANO I et al. Bundle branch reentry and the postpacing interval after entraintment by right ventricular ápex stimulation: a new approach to elucidate the mechanism of wide QRS complex tachycardia with atrioventricular dissociation. Circulation 2001; 103(8): 1102-1108

18. ISA R, MORENO M, PALAZZOLO J. Taquicardia ventricular por reentrada rama - rama en pacientes con Enfermedad de Steinert: Serie de tres casos y revisión de la literatura. Rev Chil Cardiol 2020; 39: 247-255.

19. KUMAR S, FUJII A, KAPUR S, ROMERO J, MEHTA NK, TANIGAWA $S$ et al. Beyond the Storm: Comparison of Clinical Factors, Arrhythmogenic Substrate, and Catheter Ablation Outcomes in Structural Heart Disease Patients With versus Those Without a History of Ventricular Tachycardia Storm. J Cardiovasc Electrophysiol. 2017:56-67.

20. PAPPONE C, BRUGADA J. Ablación de arritmias ventriculares en el síndrome de Brugada. Presente y futuro. Rev Esp Cardiol.2017;70(12):1046-1049.
21. QUINTO L et al. Ventricular Tachycardia Burden Reduction After Substrate Ablation: Predictors of Recurrence. Heart Rhythm 2021. doi.org/10.1016/j.hrthm.2021.02.016

22. ENRIQUEZ A, PATHAK RK, SANTANGELI P, et al. Inferior lead discordance in ventricular arrhythmias: A specific marker for certain arrhythmia locations. J Cardiovasc Electrophysiol 2017 Oct;28(10):1179-1186

23. MENG L, TSENG CH, SHIVKUMAR K, AJIJOLA O. Efficacy of Stellate Ganglion Blockade in Managing Electrical Storm: A Systematic Review JACC Clin Electrophysiol. 2017 Sep;3(9):942-949.

24. TIAN Y, WITTWER E, KAPA S, MCLEOD CH, XIAO P, NOSEWORTHY P et al. Effective Use of Percutaneous Ste1late Ganglion Blockade in Patients with Electrical Storm Circ Arrhythm Electrophysiol. 2019 Sep;12(9): e007118.

25. VASEGHI et al Cardiac Sympathetic Denervation in Patients with VT Storm. Heart Rhythm, 2014 11(3):360-6.

26. SCHWARTZ PJ, PRIORI SG, CERRONE M, SPAZZOLINI C, ODERO A, NAPOLITANO C, et al. Left Cardiac Sympathetic Denervation in the Management of High-Risk Patients Affected by the Long-QT Syndrome. Circulation. 2004; 109: 1826-33.

27. COLEMAN MA, BOS JM, JOHNSON JN, OWEN HJ, DESCHAMPS C, MOIR C, et al Videoscopic left cardiac sympathetic denervation for patients with recurrent ventricular fibrillation/malignant ventricular arrhythmia syndromes besides congenital long-QT syndrome. Circ Arrhythm Electrophysiol. 2012; 5: 782-8

28. BOURKE T, VASEGHI M, MICHOWITZ Y, SANKHLA V, SHAH M, SWAPNA N et al. Neuraxial modulation for refractory ventricular arrhythmias: value of thoracic epidural anesthesia and surgical left cardiac sympathetic denervation. Circulation. 2010;121:2255-62

29. VASEGHIR, GIMA J, KANAAN C, AJIJOLA O, MARMUREANU A, MAHAJAN A et al. Cardiac sympathetic denervation in patients with refractory ventricular arrhythmias or electrical storm: intermediate and long-term follow-up. Heart Rhythm. 2014;11

30. RICHARDSON T, LUGO R, SAAVEDRA P, CROSSLEY G, CLAIR W, SHEN S, et al. Cardiac sympathectomy for the management of ventricular arrhythmias refractory to catheter ablation. Heart Rhythm. 2018;15:56-62

31. PROCHNAU, M. HOYME. Renal denervation as a second-line option in a patient with electrical storm resistant to medical treatment and conventional radiofrequency catheter ablation. 
Journal of Electrocardiology 2018 2018;51(3):475-478

32. MAHFOUD F, MANCIA G, SCHMIEDER R, et. al. Renal Denervation in high-risk patients with hypertension. Journal of the American College of Cardiology. 2020; 75(23): 28792888

33. Townsend RR, Mahfoud F, Kandzari DE, et al. Catheter-based renal denervation in patients with uncontrolled hypertension in the absence of antihypertensive medications (SPYRAL HTN-OFF MED): a randomised, sham-controlled, proof-ofconcept trial. The Lancet. 2017; 390:2160-2170

34. KANDZARI DE, BÖHM M, MAHFOUD F, et al. Effect of renal denervation on blood pressure in the presence of antihypertensive drugs: 6-month efficacy and safety results from the SPYRAL HTN-ON MED proof-of-concept randomised trial. The Lancet. 2018 Jun 9;391(10137):2346-2355

35. LINZ D, HOHL M, ELLIOTT AD, LAU DH, MAHFOUD F, ESLER MD et al. Modulation of renal sympathetic innervation: recent insights beyond blood pressure control. Clin
Auton Res. 2018 Aug;28(4):375-384

36. TURAGAM M, WHANG W, MILLER M, NEUZIL P, ARYANA A, ROMANOV A et al. Renal Sympathetic Denervation as Upstream Therapy During Atrial Fibrillation Ablation: Pilot HFIB Studies and Meta-Analysis. JACC Clin Electrophysiol. 2021 Jan;7(1):109-123

37. Lemery R. Interventional treatment of ventricular tachycardia and electrical storm: From ablation of substrate and triggers to autonomic modulation by renal denervation. Heart Rhythm, 2014 Apr;11(4):547-8

38. REMO B, PREMINGER M, BRADFIELD J, MITTAL S, BOYLE N, GUPTA A et al. Safety and efficacy of renal denervation as a novel treatment of ventricular tachycardia storm in patients with cardiomyopathy. Heart Rhythm, 2014 Apr;11(4):541-6.

39. HAWSON, J. Renal Denervation for the Management of Refractory Ventricular Arrhythmias. A Systematic Review. et al. J Am Coll Cardiol EP. 2021;7(1): 100-8. 